\title{
Coadaptation of Prenatal and Postnatal Maternal Effects ${ }^{\star}$
}

\author{
Judith E. Lock, ${ }^{1}$ Per T. Smiseth, ${ }^{2}$ Patricia J. Moore, ${ }^{3}$ and Allen J. Moore ${ }^{3, \dagger}$
}

1. Hartpury College, University of the West of England, Hartpury House, Gloucestershire GL19 3BE, United Kingdom;

2. Faculty of Life Sciences, Michael Smith Building, University of Manchester, Oxford Road, Manchester M13 9PT, United

Kingdom;

3. School of Biosciences, University of Exeter, Cornwall Campus, Penryn TR10 9EZ, United Kingdom

Submitted February 16, 2007; Accepted June 1, 2007; Electronically published September 5, 2007

\begin{abstract}
In a wide variety of species, a female's age of first reproduction influences offspring size and survival, suggesting that there exists an optimal timing of reproduction. Mothers in many species also influence offspring size and survival after birth through variation in parental care. We experimentally separated these effects in the burying beetle Nicrophorus vespilloides to test for coadaptation between prenatal and postnatal maternal effects associated with age at first reproduction. Females that reproduced early produced offspring with lower birth weight. The amount of parental care depended on the age of first reproduction of the caretaker, as did the extent of offspring begging. As predicted for a coadaptation of maternal effects, prenatal and postnatal effects were opposite for different-aged mothers, and larval weight gain and survival was greatest when the age of the caretaker and birth mother matched. Thus, prenatal effects intrinsically associated with age of first reproduction can be ameliorated by innate plasticity in postnatal care. A coadaptation of prenatal and postnatal maternal effects may evolve to allow variable timing of the first reproductive attempt. Such a coadaptation might be particularly valuable when females are constrained from reproducing at an optimal age, as, for example, in species that breed on scarce and unpredictable resources.
\end{abstract}

Keywords: age at first reproduction, burying beetle, larval growth, maternal performance, Nicrophorus vespilloides, parental care.

\footnotetext{
* All authors collaborated to formulate the original research question and design the experiments. J.E.L. collected the data, A.J.M. and J.E.L. analyzed the data and interpreted the results, and A.J.M. wrote the article with input from all of the authors.

† Corresponding author; e-mail: a.j.moore@exeter.ac.uk.

Am. Nat. 2007. Vol. 170, pp. 709-718. (C) 2007 by The University of Chicago. 0003-0147/2007/17005-42413\$15.00. All rights reserved.

DOI: $10.1086 / 521963$
}

A mother's age at first reproduction profoundly affects offspring fitness in species from insects (Mousseau and Dingle 1991) to humans (Fraser et al. 1995; Salihu et al. 2004; Joseph et al. 2005). The effect, however, is not consistent among taxa. In vertebrates, there is often an optimal age range for reproduction. For example, in humans, mothers younger than 20 or older than 30 years old produce offspring that are smaller and have higher levels of perinatal mortality than offspring of women in their twenties, and the mortality increases with advancing age after age 30 (Joseph et al. 2005). Mothers younger than 20 years old have higher incidences of low-birth-weight babies (Fraser et al. 1995) and of neonatal death in twins (Salihu et al. 2004). Likewise, in mice, there is an optimal age at first reproduction, with young adults producing fitter offspring than adolescent or older mothers (Wang and vom Saal 2000). In birds, however, older parents produce larger eggs (Bogdanova et al. 2006) and have higher reproductive performance in general (Heidinger et al. 2006). Among insects, younger mothers tend to produce offspring that have greater juvenile survivorship (Hercus and Hoffmann 2000; Kern et al. 2001), greater longevity (Priest et al. 2002), and higher preadult performance (Fox 1993). Finally, maternal age need not influence offspring performance at all (Moore and Harris 2004).

One of the difficulties in generalizing about age-related maternal effects is that prenatal and postnatal maternal effects can be confounded in species with prolonged parental care because the quality of parental care can also depend on the age of the mother (Cameron et al. 2000; Clark et al. 2002; Weldji et al. 2006). For example, in gerbils, mothers that are older at first reproduction attend young more carefully (Clark et al. 2002). In birds, differences associated with smaller egg and hatchling weight disappear in older chicks after cross-fostering to unrelated caretakers (Bogdanova et al. 2006). The plasticity in care and response to care may reflect experience and learning (Cameron et al. 2000); it is less clear whether adaptive changes in care behavior co-vary for inexperienced mothers who vary in age at first reproduction. Maternal effects are expected to be adaptive (Mousseau and Fox 1998a, 1998b), but do different and temporally separate maternal effects evolve as an adaptive suite of characters, that is, as 
coadaptations, or does evolution influence temporally separate maternal effects independently? Answering this question requires experiments that control for confounding effects of experience and age.

Coadaptation is expected to occur when there is more than one combination of traits leading to a fitness peak. Quantitative genetic models suggest that coadapted trait expression is a common outcome when maternal effects are involved (Wolf and Brodie 1998; Wolf 2000; Kölliker 2005; Kölliker et al. 2005; Wolf and Hager 2006). This has been supported by empirical studies of parental provisioning and offspring solicitation in a variety of taxa, from insects (Agrawal et al. 2001; Lock et al. 2004) to birds (Kölliker and Richner 2001) and mammals (Hager and Johnstone 2003, 2006; Curley et al. 2004). These models and empirical studies consider coadaptation between maternal effects and offspring traits rather than between different maternal effects. However, the same reasoning should hold for maternal effects that are temporally distinct; trait combinations associated with the highest fitness should be favored. Thus, where there are trade-offs, such as might occur with age-related changes in egg quality, selection should favor the combination of pre- and postnatal maternal effects that maximizes fitness.

In this study, we experimentally examined the potential for adaptive changes in postnatal care to counter a decline in prenatal offspring quality associated with timing of reproduction by first-time mothers. We hypothesized that there could be adaptive postnatal compensation of maternal effects because timing of reproduction may be unpredictable in species where resources required for reproduction are scarce and ephemeral and competition for these resources is intense. We conducted our study in $\mathrm{Ni}$ crophorus vespilloides, a burying beetle with elaborate and essential parental care involving direct provisioning of food to offspring by regurgitation (Eggert and Müller 1997). Parental care behavior of this burying beetle is easily studied in the laboratory. Studying parental care under natural conditions while in the laboratory allows manipulative studies (e.g., Smiseth et al. 2003, 2005; Smiseth and Moore 2004b). Broods can be cross-fostered (e.g., Lock et al. 2004), as there is no kin recognition based on intrinsic characteristics of the larvae (Müller and Eggert 1990; Eggert and Müller 2000; Oldekop et al. 2007). Cross-fostering all larvae so that females never cared for their own offspring allowed us to separate prenatal and postnatal effects and control for experience and to investigate how prenatal and postnatal maternal effects interact. Importantly for this study, reproduction may occur at different ages because there is unpredictability in resources required for reproduction (Eggert and Müller 1997; Scott 1998). We measured the birth weight of newly hatched larvae to investigate how early and delayed reproduction influenced female allocation to eggs; we investigated how mothers and offspring behaved to determine the potential nature of behaviorally mediated postnatal effects on larval growth; and we examined offspring performance by measuring growth, development, and survival of larvae. As predicted for coadaptation of the sum of maternal effects, larvae do best when prenatal and postnatal influences match.

\section{Methods}

The beetles used in this experiment were the first-generation offspring of a laboratory population that originated from 110 females and 57 males. The wild-caught individuals were trapped in Japanese insect traps baited with rotting meat in Sunbank Wood, Mersey Valley, United Kingdom, in August 2003. Laboratory-bred individuals were housed individually in clear plastic containers (17 $\mathrm{cm} \times 12 \mathrm{~cm} \times 6 \mathrm{~cm})$ at $20^{\circ} \mathrm{C}$ under a $15 \mathrm{~L}: 9 \mathrm{D}$ photoperiod. The beetles were fed mealworms (Tenebrio spp.) ad lib. twice a week.

\section{Experimental Design}

We cross-fostered offspring produced by early- or delayedreproducing females to early- or delayed-reproducing caretakers in the burying beetle Nicrophorus vespilloides to experimentally separate the consequences of prenatal and postnatal maternal effects. Reproduction by N. vespilloides differs from most insects in that parents (female alone, male alone, or both parents together) directly provision offspring with predigested food through regurgitation (Eggert and Müller 1997; Scott 1998). Females (or males) locate a vertebrate carcass, prepare the carcass by stripping furs, scales, or feathers, and roll the carcass into a ball that is wholly or partially buried. Males without partners attract females using a pheromone and then cooperate with the females in carcass preparation. Females, if mated, can bury a carcass by themselves and are as successful at reproduction as cooperating pairs. Eggs are laid off of the carcass in the nearby soil. Once the eggs hatch, the larvae crawl to the carcass and enter the crater formed by the parents on the top of the ball. Larvae beg to parents by waving their forelegs and touching their parents' mouthparts. Begging and direct provisioning is greatest during the first 48 $\mathrm{h}$, after which the larvae obtain food primarily by selffeeding (Smiseth et al. 2003). The carcass is the sole food resource for the developing larvae. Parental care involves keeping the carcass free of fungus and bacteria with anal secretions as well as partially digesting the carcass and feeding the offspring directly (Smiseth and Moore 2004a). This continues until the larvae reach the final instar, approximately $72-96 \mathrm{~h}$ after hatching under our laboratory conditions. Approximately 48-96 h after the larvae reach 
the final instar, both the mother and the larvae disperse from the carcass (Lock et al. 2004). Once larvae have dispersed from the carcass, they do not feed again until after they have pupated into the adult form. Female sexual maturity is reached around 2 weeks after eclosion to adult.

We separated prenatal and postnatal maternal effects experimentally by controlling timing of reproduction of the mother and measuring offspring traits immediately after hatching, before receiving any care, and at dispersal after having received care and experiencing the environment provided by a caretaker. We manipulated the age of reproduction of the caretaker (caretaker treatment) and age of reproduction of the dam (offspring treatment) by allowing females to breed at either 2 weeks or 6 weeks after eclosion to adulthood. Burying beetles breed only if provided with a vertebrate carcass that is sufficiently large. In order to ensure the timing of cross-fostered broods, multiple females were bred simultaneously. Thus, not all females were used as either donor mothers or caretakersthat is, there was no attempt to have reciprocal crossfostering as in some other studies (Cheverud 1984; Lock et al. 2004). In the offspring treatment, there were "early offspring" and "delayed offspring," which reflects the treatment of the larvae's biological mother and describes the source of prenatal maternal effect. In the caretaker treatment, there were "early mothers" and "delayed mothers," which reflects the reproductive experience of the females providing care and describes the source of postnatal maternal effects.

Eggs were removed from the soil around the prepared carcass, hatched in petri dishes, and cross-fostered to unrelated experimental caretakers who had also laid eggs at the same time, so that no female in the study cared for her own offspring. The female and the carcass were removed to a new box with fresh soil to avoid late-hatched eggs arriving at the carcass. Petri dishes were checked regularly throughout the day for hatched larvae to ensure that all offspring were of similar age. All larvae that were used had hatched within a few hours of each other. Clutch size was standardized to $8-10$ (average $=9.3$ ). Carcass size was also standardized, with carcasses ranging from 10.3 to 14.5 $\mathrm{g}$ (average $=12.1 \mathrm{~g})$. We controlled these factors because burying beetle females regulate the number of eggs they lay based on the size of the carcass (Müller et al. 1990) and use filial cannibalism to reduce their brood to a size that fits the carcass (Bartlett 1987). Offspring weight is a function of brood size (Bartlett 1987), as is the amount of begging by offspring (Smiseth and Moore 2002). We therefore standardized brood and carcass sizes to a range of sizes where the relationship between number of offspring in a brood and carcass size are unrelated (Smiseth and Moore 2002) to ensure equivalent experiences for fe- males and equivalent starting points for all broods. We saw no evidence of cannibalism in any of our broods.

\section{Prenatal Maternal Effects}

Females mature and lay eggs only when provided with a carcass that can be used as a food source for offspring (Trumbo 1997; Scott et al. 2005). We manipulated age at first reproduction by randomly assigning females to two treatments: early reproduction at 2 weeks after adult eclosion $(N=36$; immediately following reproductive maturation) and delayed reproduction at 6 weeks after adult eclosion $(N=39)$. We lack birth weight for four samples of the early reproduction treatments. Given the ephemeral nature of the resource required for reproduction, we chose these periods as extremes of potential natural variation. Two weeks is the earliest a female could reproduce, but reproduction at this age is unlikely because the resources necessary for reproduction are scarce and unpredictable. Six weeks represents approximately half or two-thirds of a breeding season in a year and, therefore, sufficient time for females to discover the necessary resources and a period well before reproductive senescence.

Females were provided with a thawed mouse carcass (Livefoods Direct, Sheffield, U.K.) at 5 p.m., 2 h before the subjective night in the laboratory and mimicking dusk, when $N$. vespilloides in the wild typically search for carrion (Eggert 1992). Sixty hours later, when eggs were observed in the soil, the female and the carcass were moved to a new box with fresh soil. Eggs were collected and placed in a petri dish on damp filter paper until they hatched.

We measured the weight of the entire brood before giving it to the caretaker, to obtain an average birth weight for the larvae. This was done to determine whether there were prenatal effects. Weight at hatching provides a measure of prenatal maternal expenditure, as eggs were laid away from the carcass and larvae had no contact with the caretaker or any food source before they were weighed.

\section{Postnatal Maternal Effects}

For this experiment, behavioral observations of parental care and offspring begging were carried out $24 \mathrm{~h}$ after each caretaker was given her brood. Parental and larval behaviors were recorded using instantaneous scan sampling (Martin and Bateson 1986). Observations lasted $30 \mathrm{~min}$, during which all behaviors performed by parents and offspring were scored as in previous studies (Smiseth et al. 2003, 2005; Lock et al. 2004; Smiseth and Moore 2004a). We analyzed two parental care behaviors directed specifically toward the offspring and known to influence offspring fitness under normal conditions (Lock et al. 2004): (1) percentage of time the caretaker spent in the cavity in 


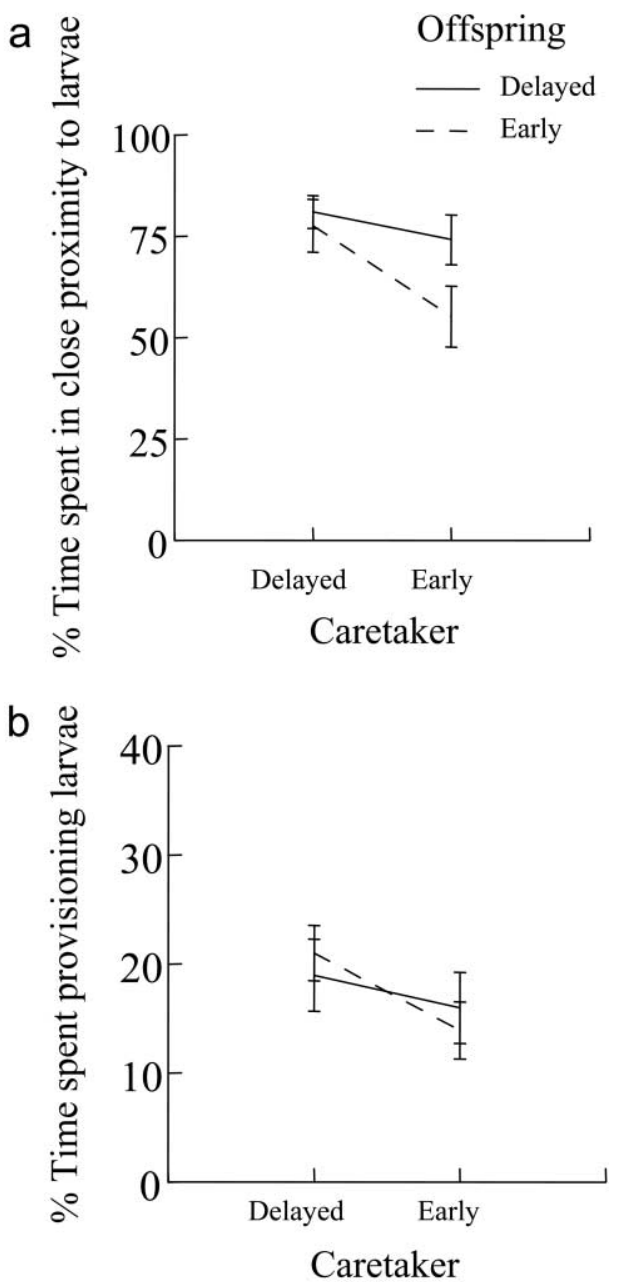

Figure 1: Changes in care behavior of caretakers associated with their age of reproduction or the age of reproduction of the mothers of the larvae. Data shown as means \pm SE. Offspring may be "early" or "delayed," reflecting the treatment of the larvae's biological mother and the timing of her reproduction, and the terms describe the source of prenatal maternal effect. A caretaker may be "early" or "delayed," reflecting the reproductive timing of the females providing care, and the terms describe the source of postnatal maternal effects. $a$, Proportion of time the female spent in the cavity without provisioning larvae with food, behavior that has negative effects on offspring fitness (Lock et al. 2004). There is a significant main effect of the caretaker treatment but no significant effect of the offspring treatment or the interaction between caretaker and offspring treatments. $b$, Proportion of time females spent provisioning larvae with food when in close proximity, behavior that has a positive effect on offspring fitness (Lock et al. 2004). There is a significant main effect of the caretaker treatment but no significant effect of the offspring treatment or the interaction between caretaker and offspring treatments.

close proximity to the offspring (i.e., within one pronotum length of the offspring) without feeding the offspring, and (2) percentage of time the caretaker spent directly provisioning the offspring, indicated by mouth-to-mouth contact between parent and larva. This former measure is negatively related to offspring fitness while the latter is positively related to offspring fitness (Lock et al. 2004). We measured two behaviors indicating offspring begging effort: (1) the average percent of time spent begging when the parent was present and in close proximity, that is, measure of begging effort by each larva; and (2) the mean number of larvae begging in scans where at least one larva was begging, that is, a measure of sibling competition for food from the parent (Lock et al. 2004; Smiseth and Moore 2004a). The data were log transformed to achieve a normal distribution and analyzed by $2 \times 2$ ANOVA using JMP Professional, version 5.

\section{Combined Maternal Effects}

We used the early and delayed breeding design described above in a fully factorial experiment to investigate the joint and separate influences of prenatal and postnatal maternal effects. We measured the effects of our treatments on offspring development and larval weight gain, using two-way ANOVA. Development was measured, as in other studies (Lock et al. 2004), as the sum of the time larvae spent (1) on a carcass (from birth to dispersal from the carcass), (2) as "wandering" larvae (off the carcass and nonfeeding), and (3) as pupae. Developmental stages were checked daily. Weight gain was measured as the change in weight from birth to dispersal from the carcass. All larvae that dispersed from the carcass were weighed, as were eclosing adults. Although development times and weight gain were measured on individuals, family means were used to avoid problems of pseudoreplication. We analyzed the relationship between these traits and absolute offspring fitness, measured as survival to adult (no $=0$, yes $=1$ ), using logistic regression in JMP Professional. Relationships between birth weight (representing prenatal maternal allocation) and weight gain (reflecting postnatal maternal allocation) and final weight at eclosion were investigated by Pearson correlations. We also investigated the relationship between initial weight and duration of different development stages.

Coadapted traits generally show negative relationships (Agrawal et al. 2001), although this also depends on the targets of selection when both parents and offspring traits are involved (see Kölliker et al. 2005). Since we investigated only maternal effects in this study, the target is the same individual (the mother), and we therefore expect negative correlations between early and late maternal effects. Where possible, where females both provided a clutch to be fostered and cared for a foster clutch (see above), we investigated potential coadaptations by examining correlations between the weight gain of fostered broods and birth weight of the caretakers' own larvae. We examined these 
a
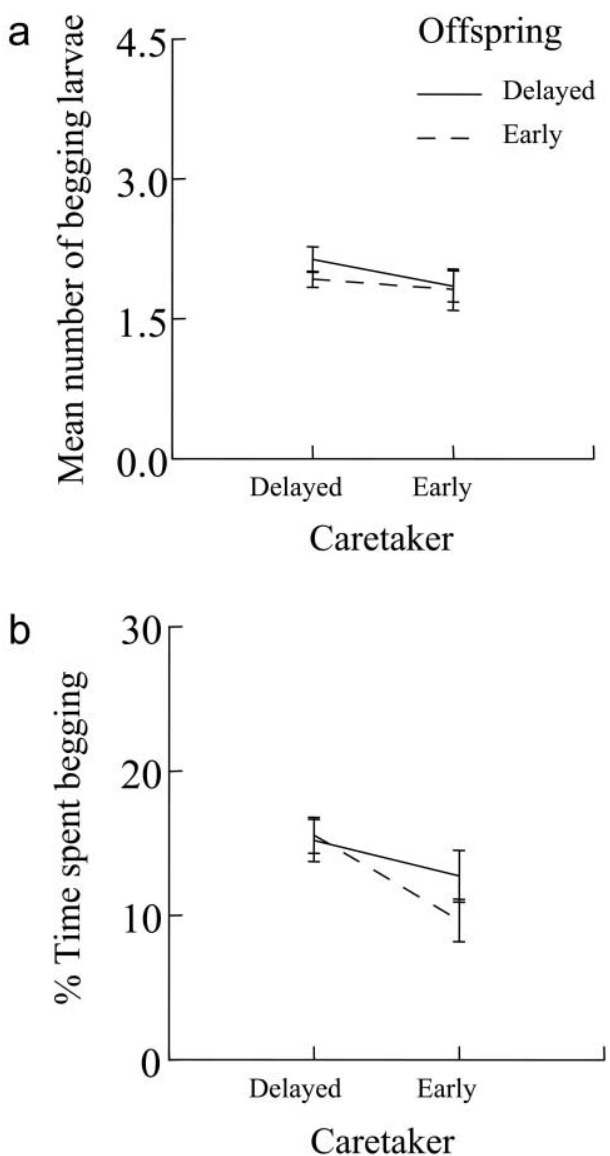

Figure 2: Changes in offspring begging behavior associated with age of reproduction of their mothers or their caretakers. Data shown as means \pm SE with treatments as in figure 1. $a$, Mean number of offspring begging within a brood, a measure of offspring conflict negatively associated with offspring fitness (Lock et al. 2004). Neither of the main effects nor their interaction are statistically significant. $b$, Average proportion of time a larva spent begging, a behavior positively associated with offspring fitness (Lock et al. 2004). There is a significant main effect of the caretaker treatment but no significant effect of the offspring treatment or the interaction between caretaker and offspring treatments.

both within each treatment and overall, as the prediction is that treatment should not affect this relationship because there is no kin recognition based on intrinsic characteristics of the larvae (Müller and Eggert 1990; Eggert and Müller 2000).

\section{Results}

\section{Prenatal Maternal Effects}

Age at first reproduction influenced allocation of resources to eggs. Offspring from different-aged mothers had significantly different birth weights $(F=4.633, \mathrm{df}=1,69$,
$P=.035$ ), with newborn offspring produced by females with delayed reproduction being significantly heavier $(\bar{X}=2.88 \mathrm{mg}, \mathrm{SE}=0.11)$ than offspring produced by early-reproducing females $(\bar{X}=2.59 \mathrm{mg}, \mathrm{SE}=0.07)$. There was no significant association between the age of the caretaker and weight of the foster brood $(F=0.434$, $\mathrm{df}=1,69, P=.496)$, indicating that broods were randomly allocated to treatments.

\section{Postnatal Maternal Effects}

Age at first reproduction influenced maternal behavior (fig. 1). Caretakers with delayed reproduction spent significantly more time in the cavity without feeding the offspring than did caretakers with early reproduction $(F=$ 5.099 , $\mathrm{df}=1,71, P=.003$; fig. $1 A)$. In contrast, the offspring treatment did not have a significant influence on the behavior of the caretaker $(F=1.012, \mathrm{df}=1,71$, $P=.318)$, and there was no significant interaction between caretaker and offspring treatment on the time spent in the cavity $(F=1.258, \mathrm{df}=1,71, P=.266)$.

The age at first reproduction had a similar influence on female provisioning of the offspring at $24 \mathrm{~h}$ of age (fig. $1 B)$. Females with delayed reproduction spent more time provisioning the larvae $(F=3.805, \mathrm{df}=1,71, P=.05)$. Again, there was no significant effect of offspring treatment ( $F=0.006, \mathrm{df}=1,71, P=.940)$ on amount of time spent by the caretaker provisioning the larvae or a significant interaction between caretaker and offspring treatment ( $F=0.994, \mathrm{df}=1,71, P=.322)$ on amount of time spent by the caretaker provisioning the larvae.

Changes in maternal behavior reflected differences associated with the females rather than responses to cues provided by the offspring (fig. 2). We found no significant effect of caretaker treatment $(F=1.826, \mathrm{df}=1,71$, $P=.181)$, offspring treatment $(F=0.685, \mathrm{df}=1,71$, $P=.411)$, or their interaction $(F=0.354, \mathrm{df}=1,71$, $P=.554)$ on the mean number of larvae begging in a brood (fig. $2 A$ ). In contrast, the average percent of time a larva spent begging was affected by the caretaker treatment $(F=9.854$, df $=1,71, P=.002)$, with offspring begging more to females with delayed reproduction (fig. $2 B$ ). This reflects the change in caretaker behavior because offspring beg more when females spend more time in close proximity to offspring. There is a highly significant correlation between the percent of time spent begging by a larva and the percent of time spent provisioning by a caretaker $(r=0.77, n=75, P<.001)$. Further support for a response of the offspring to the maternal environment rather than to intrinsic influences is found in the lack of significant differences in the average amount of time a larva spent begging associated with the offspring 
treatment $(F=1.012$, df $=1,71, P=.318)$ or the interaction between caretaker and offspring treatment $(F=$ 1.258, df $=1,71, P=.266)$.

\section{Combined Maternal Effects}

The interaction between prenatal and postnatal maternal effects influenced offspring growth and offspring development (fig. 3). Offspring increased their weight 100-fold, from an average of $2.7 \mathrm{mg}$ at birth to an average of 208.0 $\mathrm{mg}$ at adult eclosion, in an average 6.6 days of feeding. There was no significant main effect on weight gain from birth to dispersal from the carcass. Neither the offspring treatment $(F=0.016$, df $=1,67, P=.900)$ nor the caretaker treatment $(F=0.117, \mathrm{df}=1,67, P=.733)$ was associated with variation in the amount of weight larvae gained on the carcass. However, there was a significant effect of the interaction between the timing of reproduction by the caretakers and the offspring treatment on the amount of weight gained $(F=6.044, \mathrm{df}=1,67, P=$ $.017)$. The heaviest offspring were produced when the offspring treatment and the caretaker treatment matched (fig. $3 A)$. Average weight gain was significantly correlated with average weight at eclosion $(r=0.86, P<.001)$, with nonsignificant correlations between average initial weight and average weight gain $(r=0.16, N=71, P=.172)$ and average initial weight and average eclosion weight $(r=$ $0.14, N=71, P=.250$ ).

Age of first reproduction also influenced the number of days offspring took to develop from egg to adulthood in a similar pattern (fig. $3 B$ ). Although for this trait there was a marginally nonsignificant effect of offspring treatment on the length of development from birth to adult eclosion $(F=3.723$, df $=1,71, P=.058)$, caretaker treatment did not approach significance $(F=0.110$, $\mathrm{df}=1,71, P=.741)$. Again, there was a highly significant effect of the interaction between offspring and caretaker treatment on offspring development $(F=8.972$, df $=$ $1,71, P=.004)$. Development took longest when offspring and caretaker mating treatments matched (fig. $3 B$ ). The length of development was weakly correlated with weight at hatching $(r=0.21, n=71, P=.085)$ and significantly correlated with eclosion weight $(r=0.35$, $n=71, P=.002)$ and weight gain $(r=0.42, n=71$, $P=.0003)$.

Not all offspring that were born and placed with a mother survived to adulthood. Offspring that were heavier at birth had higher survivorship (logistic regression; Wald $\left.\chi^{2}=4.470, \mathrm{df}=1, P=.035\right)$. Individuals that gained more weight as larvae were also more likely to survive to adulthood (logistic regression; Wald $\chi^{2}=12.709$, $\mathrm{df}=$ $1, P<.001)$.

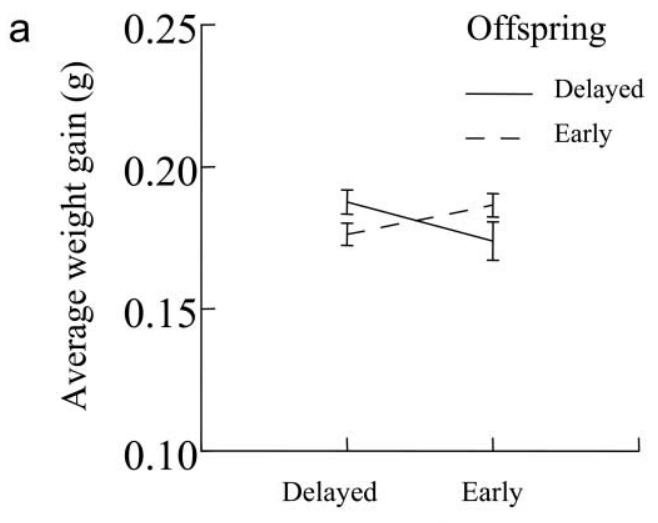

Caretaker

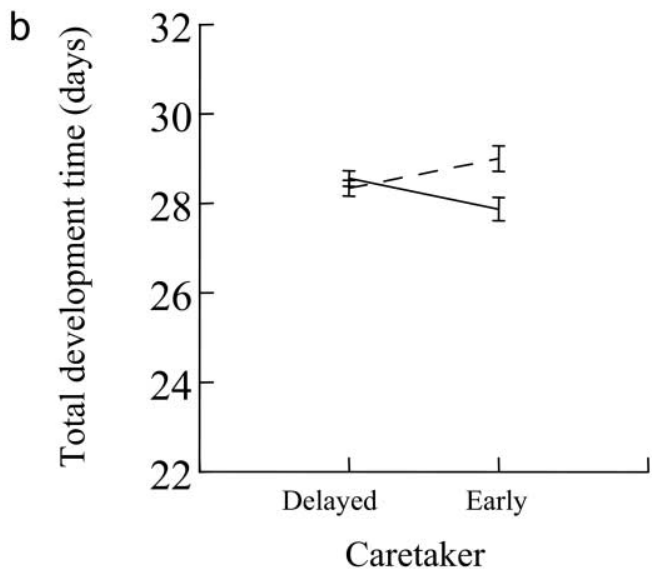

Figure 3: Changes in offspring performance associated with age of reproduction of their mothers or their caretakers. Data shown as means $\pm \mathrm{SE}$ with treatments as in figure 1. $a$, Influence on larval growth in the presence of the caretaker and while receiving care. Neither main effect is significant, but there is a highly significant effect of the interaction between treatments. $b$, Influence on offspring development. There is no significant effect of caretaker treatment, and there is a marginal effect of offspring treatment, but there is a significant effect of the interaction between the two treatments.

Correlations between the birth weights of the females' own larvae and the weight gain of cross-fostered larvae support a coadaptation. All such correlations were negative: older mothers caring for larvae from older mothers $(r=$ -0.07, $n=16, P=.400$ ), older mothers caring for larvae of younger mothers $(r=-0.70, n=13, P=.004)$, younger mothers caring for offspring of older mothers ( $r=-0.69, n=12, P=.006)$, and younger mothers caring for offspring of younger mothers $(-0.12, n=13$, $P=.348)$. The overall relationship between the birth weight of larvae and the weight gain of foster larvae was negative and significantly different from zero $(r=-0.30$, $n=54, P=.015)$. 


\section{Discussion}

As in many organisms, timing of first reproduction influenced offspring quality in the burying beetle Nicrophorus vespilloides. Both prenatal and postnatal maternal effects changed with the mother's age. Females that reproduced as soon as they were sexually mature did not produce eggs as large as those of females who delayed reproduction by a few weeks. However, age-related differences in postnatal maternal effects ameliorated any effects that might have been caused by age-related differences in prenatal maternal effects. If females that reproduced early cared for offspring from mothers that reproduced early, they reared offspring as large as those produced and cared for by females that delayed reproduction. Only when offspring and mother treatments did not match were smaller offspring produced. Our research shows that when there is an opportunity for both prenatal and postnatal effects because of parental care, females can adjust their behavior so that the size of the offspring is unaffected by variation in timing of reproduction. This adjustment is made without learning, experience, or determination of offspring need. Thus, our study shows that prenatal and postnatal effects co-vary in $N$. vespilloides. This appears to be an evolved response, because the combined prenatal and postnatal maternal effects ultimately had the greatest influence on offspring fitness in $N$. vespilloides and therefore appear to be coadapted. The implication of our finding is that documenting the effects of aging on reproduction in species with elaborate parental care may be more complicated than has been appreciated. It is important to consider the sum of all maternal effects, prenatal and postnatal, when considering age-related changes in reproduction.

In studies that aim to determine the adaptive nature of maternal effects, it is important to measure fitness effects directly rather than infer fitness. For example, it is not always obvious how egg size relates to offspring quality; it is not simply a case of bigger always being better (Fox and Czesak 2000). The composition of eggs may be more important than the total size (Fox and Czesak 2000; Giron and Casas 2003). Furthermore, there may be interactions with environments after hatching (Mousseau and Fox $1998 b$ ), and the relationship can be as complicated as survival depending on a match between egg size and resources (Fox and Mousseau 1996). In seed beetles, maternal effects are also plastic and adaptive as females vary egg size to match specific host plants, ensuring the greatest survivorship (Fox et al. 1997). In our study, higher birth weight (reflecting a larger egg size) was associated with greater chance of survival to adulthood. We also found that greater weight gain during the period of parental care resulted in a greater chance of survival. We found this positive relationship between size at dispersal and survival in a pre- vious study as well (Lock et al. 2004). However, individuals can be large at the time of dispersal either by starting large or by gaining the most weight throughout the period of parental care, and these effects trade off so that there is a coadaptation between birth weight and larval weight gain.

It is also difficult to infer specifics regarding the exact mechanism for how changes in parental investment underlie the postnatal maternal effect from our study. Parents can influence their offspring in a variety of ways after their birth (Mousseau and Fox 1998a, 1998b). Quantitative geneticists have long known that it is easier to quantify maternal performance (the effect of mothers on their offspring) than to measure specific mechanisms underlying maternal effects (Cheverud and Moore 1994, pp. 72-73). Behavioral biologists have also recognized that it can be more accurate to measure offspring performance, such as weight gain, as a surrogate for maternal allocation. Specific behavioral measures, such as suckling in mammals, for example, do not always reflect the allocation of resources or parental investment (Cameron 1998). We measured some of the likely postnatal behavioral changes by mothers but do not suggest that we have captured the exact or entire mechanisms of maternal adaptations. We found that older females spent more time in the cavity without feeding offspring, which in unmanipulated broods has a negative influence on fitness because of competition with the offspring for food (Lock et al. 2004). Mothers feed themselves and their offspring from the carcass. However, we also found that older mothers provisioned offspring more during the observation period in which we measured this behavior.

There are several reasons why the provisioning measured in this study may not accurately reflect postnatal maternal allocation of resources. First, the amount of begging and the amount of provisioning changes over time in burying beetles (Smiseth et al. 2003). Optimal levels of maternal care may depend as much on the timing of care as on the amount (Cameron et al. 2000) and on minimizing parent-offspring conflicts (Lock et al. 2004). In addition, there are a number of ways females influence the larval environment in addition to direct provisioning of food by regurgitation. Mothers also prepare and tend to the carcass, and the quality of this maintenance behavior may also influence developing young. Females are not necessarily feeding from the carcass when they are in the crypt; they may be preparing it for ease of access by the larvae. It may be that female age and the ability to prepare a carcass co-vary even though all the females in this study were naive and virgin. Older females may put more effort into carcass preparation and maintenance at the expense of very early provisioning. Unfortunately, we do not have measures of provisioning over time. We observed provisioning at the time period found to be critical in our other 
studies-but these studies were conducted on young females. Thus, we cannot resolve from this study the specific qualities that make for a good mother, other than to note that the quality of mothering depended on the needs of the offspring. Because we experimentally separated prenatal and postnatal effects and could examine the interaction between the two, our research supports a clear coadaptation between the overall effects of the two stages of maternal effects independent of experience and learning. The changes in maternal effects with age are adaptive and specific.

Our study does, however, suggest a number of areas for further consideration. Evolutionary theory suggests that maternal effects are an effective mechanism to counter variable environments and, therefore, can be adaptive (Mousseau and Fox 1998a, 1998b). However, the quality of maternal effects also can be influenced by the age of the female, resulting in an optimal age at first reproduction. Timing of female reproduction influences offspring fitness (Fraser et al. 1995; Wang and vom Saal 2000; Salihu et al. 2004; Joseph et al. 2005). Aging of mothers also correlates with a decrease in offspring performance (Mousseau and Dingle 1991; Fox 1993; Hercus and Hoffmann 2000; Kern et al. 2001; Priest et al. 2002). These effects, however, are all prenatal. In species where mothers care for their offspring after birth, it is possible for females of different ages to adjust their care behavior (Cameron et al. 2000; Clark et al. 2002). Our research shows that different maternal effects must be considered together, because prenatal and postnatal effects may not be additive. Thus, evolution may act to ensure that prenatal and postnatal effects match so as to optimize offspring fitness. We suggest this is particularly likely in species where elaborate parental care of young occurs and where the timing of reproduction is uncertain due to scarcity and unpredictable distribution of required resources.

Evolutionary theory also suggests that females should change their investment in offspring, investing more in offspring with age (Trivers 1972; Pianka and Parker 1975; Clutton-Brock 1984, 1991). This theory, however, neglects age-related maternal effects (Bernardo 1996; Cameron et al. 2000). This neglect is perhaps not surprising given that maternal effects have only recently been considered in studies of aging (Kern et al. 2001; Priest et al. 2002). However, incorporating maternal effects into life-history theories of reproduction may be difficult, because there are few generalizations for the relationship between maternal age and offspring fitness. While there is a general trend for older mothers to produce offspring with lower fitness (Fox 1993; Hercus and Hoffmann 2000; Kern et al. 2001; Priest et al. 2002) and for these effects to persist over multiple generations (Mousseau and Dingle 1991; Mousseau and Fox 1998b; Hercus and Hoffmann 2000), it is also possible that age-related declines can be ameliorated by "targeted reproductive effort" (Cameron et al. 2000). Targeted reproductive effort occurs when there is no difference in the overall investment among mothers but when mothers differ in the timing of resource allocation. Care or resource allocation is targeted to the time when it is most valuable. Targeted reproductive effort may be especially valuable if, as in this study, postnatal maternal effects can trade off or compensate prenatal maternal effects. Targeting may occur because of better experience or because the potential for future reproductive effort changes with age. Thus, incorporating a consideration of age-related maternal effects in evolutionary theories of aging and optimal reproduction will require a knowledge and consideration of the potential suite of maternal effects for individual species. Species with extensive parental care may respond differently from those that are limited to prenatal maternal effects.

Maternal age and condition can influence both prenatal and postnatal maternal effects, and both can have important influences on evolution because there can be both positive and negative maternal effects. Thus, there is an opportunity to compensate for age and environmentally induced differences among females if prenatal and postnatal effects respond in opposite patterns. An evolutionary coadaptation between prenatal and postnatal maternal effects can therefore be an evolved mechanism to overcome unpredictable timing of first reproduction, especially in species with parental care. Our research supports such a coadaptation. However, the potential influence and coadaptation of maternal effects has yet to be incorporated into theories of age-related reproduction.

\section{Acknowledgments}

This research was supported by a Natural Environment Research Council (NERC) postgraduate fellowship to J.E.L. and NERC grants to A.J.M. and P.T.S. We thank M. Gibbs and S. Musa for laboratory assistance with this project and D. Hosken, C. House, L. Rowe, C. Stamper, C. Walling, and two anonymous reviewers for constructive comments on the manuscript.

\section{Literature Cited}

Agrawal, A. F., E. D. Brodie III, and J. Brown. 2001. Parent-offspring coadaptation and the dual genetic control of maternal care. Science 292:1710-1712.

Bartlett, J. 1987. Filial cannibalism in burying beetles. Behavioral Ecology and Sociobiology 21:179-183.

Bernardo, J. 1996. The particular maternal effect of propagule size, especially egg size: patterns, models, quality of evidence and interpretations. American Zoologist 36:216-236.

Bogdanova, M. I., R. G. Nager, and P. Monaghan. 2006. Does parental 
age affect offspring performance through differences in egg quality? Functional Ecology 20:132-141.

Cameron, E. Z. 1998. Is suckling behaviour a useful predictor of milk intake? a review. Animal Behaviour 56:521-532.

Cameron, E. Z., W. L. Linklater, K. J. Stafford, and E. O. Minot. 2000. Aging and improving reproductive success in horses: declining residual reproductive value or just older and wiser? Behavioral Ecology and Sociobiology 47:243-249.

Cheverud, J. M. 1984. Evolution by kin selection: a quantitative genetic model illustrated by maternal performance in mice. Evolution 38:766-777.

Cheverud, J. M., and A. J. Moore. 1994. Quantitative genetics and the role of the environment provided by relatives in behavioral evolution. Pages 67-100 in C. R. B. Boake, ed. Quantitative genetic studies of behavioral evolution. University of Chicago Press, Chicago.

Clark, M., M. Moghaddas, and B. G. Galef Jr. 2002. Age at first mating affects parental effort and fecundity of female Mongolian gerbils. Animal Behaviour 63:1129-1134.

Clutton-Brock, T. H. 1984. Reproductive effort and terminal investment in iteroparous animals. American Naturalist 123:212229.

1991. Lifetime data and the measurement of selection. Evolution 45:454.

Curley, J. P., S. Barton, A. Surani, and E. B. Keverne. 2004. Coadaptation in mother and infant regulated by a paternally expressed imprinted gene. Proceedings of the Royal Society B: Biological Sciences 271:1303-1309.

Eggert, A.-K. 1992. Alternative male mate finding tactics in burying beetles. Behavioral Ecology 3:243-254.

Eggert, A.-K., and J. K. Müller. 1997. Biparental care and social evolution in burying beetles: lessons from the larder. Pages 216236 in J. C. Choe and B. J. Crespi, eds. The evolution of social behavior in insects and arachnids. Cambridge University Press, Cambridge.

- 2000. Timing of oviposition and reproductive skew in cobreeding female burying beetles (Nicrophorus vespilloides). Behavioral Ecology 11:357-366.

Fox, C. W. 1993. The influence of maternal age and mating frequency on egg size and offspring performance in Callosobruchus maculatus (Coleoptera: Bruchidae). Oecologia (Berlin) 96:139-146.

Fox, C. W., and M. E. Czesak. 2000. Evolutionary ecology of progeny size in arthropods. Annual Review of Entomology 45:341-369.

Fox, C. W., and T. A. Mousseau. 1996. Larval plant affects fitness consequences of egg size variation in the seed beetle Sator limbatus. Oecologia (Berlin) 107:541-548.

Fox, C. W., M. S. Thacker, and T. A. Mousseau. 1997. Egg plasticity in a seed beetle: an adaptive maternal effect. American Naturalist 149:149-163.

Fraser, A. M., J. E. Brockert, and R. H. Ward. 1995. Association of young maternal age with adverse reproductive outcomes. New England Journal of Medicine 332:1113-1117.

Giron, D., and J. Casas. 2003. Mothers reduce egg provisioning with age. Ecology Letters 6:273-277.

Hager, R., and R. Johnstone. 2003. The genetic basis of family conflict resolution in mice. Nature 421:491-492.

- 2006. The influence of phenotypic and genetic effects on maternal provisioning and offspring weight gain in mice. Biology Letters 2:81-84.

Heidinger, B. J., I. C. T. Nisbet, and E. D. Ketterson. 2006. Older parents are less responsive to a stressor in a long-lived seabird: a mechanism for increased reproductive performance with age? Proceedings of the Royal Society B: Biological Sciences 273:2227-2231. Hercus, M. J., and A. A. Hoffmann. 2000. Maternal and grandmaternal age influence offspring fitness in Drosophila serrata. Proceedings of the Royal Society B: Biological Sciences 267:2105-2110. Joseph, K. S., A. C. Allen, L. Dodds, L. A. Turner, H. Scott, and R. Liston. 2005. The perinatal effects of delayed childbearing. Obstetrics and Gynecology 105:1410-1418.

Kern, S., M. Ackermann, S. C. Stearns, and T. J. Kawecki. 2001. Decline in offspring viability as a manifestation of aging in Drosophila melanogaster. Evolution 55:1822-1831.

Kölliker, M. 2005. Ontogeny in the family. Behavior Genetics 35:718.

Kölliker, M., and H. Richner. 2001. Parent-offspring conflict and the genetics of offspring solicitation and parental response. Animal Behaviour 62:395-407.

Kölliker, M., E. D. Brodie III, and A. J. Moore. 2005. The coadaptation of parental supply and offspring demand. American Naturalist 166:506-516.

Lock, J. E., P. T. Smiseth, and A. J. Moore. 2004. Selection, inheritance, and the evolution of parent-offspring interactions. American Naturalist 164:13-24.

Martin, P., and P. Bateson. 1986. Measuring behaviour: an introductory guide. Cambridge University Press, Cambridge.

Moore, P. J., and W. E. Harris. 2004. Is a decline in offspring quality a necessary consequence of maternal ageing? Proceedings of the Royal Society B: Biological Sciences 270(suppl.):192-194.

Mousseau, T. A., and H. Dingle. 1991. Maternal effects in insect life histories. Annual Review of Entomology 36:511-534.

Mousseau, T. A., and C. W. Fox. 1998a. The adaptive significance of maternal effects. Trends in Ecology \& Evolution 13:403-407.

- eds. 1998b. Maternal effects as adaptations. Oxford University Press, Oxford.

Müller, J. K., and A.-K. Eggert. 1990. Time-dependent shifts between infanticidal and parental behavior in female burying beetles: a mechanism of indirect mother-offspring recognition. Behavioral Ecology and Sociobiology 27:11-16.

Müller, J. K., A.-K. Eggert, and E. Furlkröger. 1990. Clutch size regulation in the burying beetle Necrophorus vespilloides Herbst (Coleoptera: Silphidae). Journal of Insect Behavior 3:265-270.

Oldekop, J. A., P. T. Smiseth, H. D. Piggins, and A. J. Moore. 2007. Adaptive switch from infanticide to parental care: how do beetles time their behaviour? Journal of Evolutionary Biology 20:19882004, doi:10.1111/j.1420-9101.2007.01364.x.

Pianka, E. R., and W. S. Parker. 1975 Age-specific reproductive tactics. American Naturalist 109:453-464.

Priest, N. K., B. Mackowiak, and D. E. L. Promislow. 2002. The role of parental age effects on the evolution of aging. Evolution 56: 927-935.

Salihu, H. M., D. Emusu, M. H. Aliyu, R. S. Kirby, and G. R. Alexander. 2004. Low maternal age and neonatal survival of extremely preterm twins (20-28 weeks of gestation). Obstetrics and Gynecology 103:1248-1254.

Scott, M. P. 1998. The ecology and behavior of burying beetles. Annual Review of Entomology 43:595-618.

Scott, M. P., S. C. Panaitof, and K. L. Carleton. 2005. Quantification of vitellogenin-mRNA during maturation and breeding of a burying beetle. Journal of Insect Physiology 51:323-331.

Smiseth, P. T., and A. J. Moore. 2002. Does resource availability affect 
offspring begging and parental provisioning in a partially begging species? Animal Behaviour 63:577-585.

. 2004a. Behavioral dynamics between caring males and females in a beetle with facultative biparental care. Behavioral Ecology 15:621-628.

2004b. Signalling of hunger when offspring forage by both begging and self-feeding. Animal Behaviour 67:1083-1088.

Smiseth P. T., C. T. Darwell, and A. J. Moore. 2003. Partial begging: an empirical model for the early evolution of offspring signalling. Proceedings of the Royal Society B: Biological Sciences 270:17731777.

Smiseth, P. T., C. Dawson, E. Varley, and A. J. Moore. 2005. How do caring parents respond to mate loss? differential response by males and females. Animal Behaviour 69:551-559.

Trivers, R. L. 1972. Parental investment and sexual selection. Pages 136-179 in B. Campbell, ed. Sexual selection and the descent of man, 1871-1971. Aldine, Chicago.

Trumbo, S. T. 1997. Juvenile hormone-mediated reproduction in burying beetles: from behavior to physiology. Archives of Insect Biochemistry and Physiology 35:479-490.

Wang, M.-H., and F. S. vom Saal. 2000. Maternal age and traits in offspring. Nature 407:469-470.

Weldji, R. B., J.-M. Gaillard, N. G. Yoccoz, O. Holand, A. Mysterud, A. Loison, M. Nieminen, and N. C. Stenseth. 2006. Good reindeer mothers live longer and become better in raising offspring. Proceedings of the Royal Society B: Biological Sciences 273:1239-1244.

Wolf, J. B. 2000. Gene interactions from maternal effects. Evolution 54:1882-1898.

Wolf, J. B., and E. D. Brodie III. 1998. The coadaptation of parental and offspring characters. Evolution 52:299-308.

Wolf, J. B., and R. Hager. 2006. A maternal-offspring coadaptation theory for the evolution of genomic imprinting. PLoS Biology 4: e380, doi:10.1371/journal.pbio.0040380.

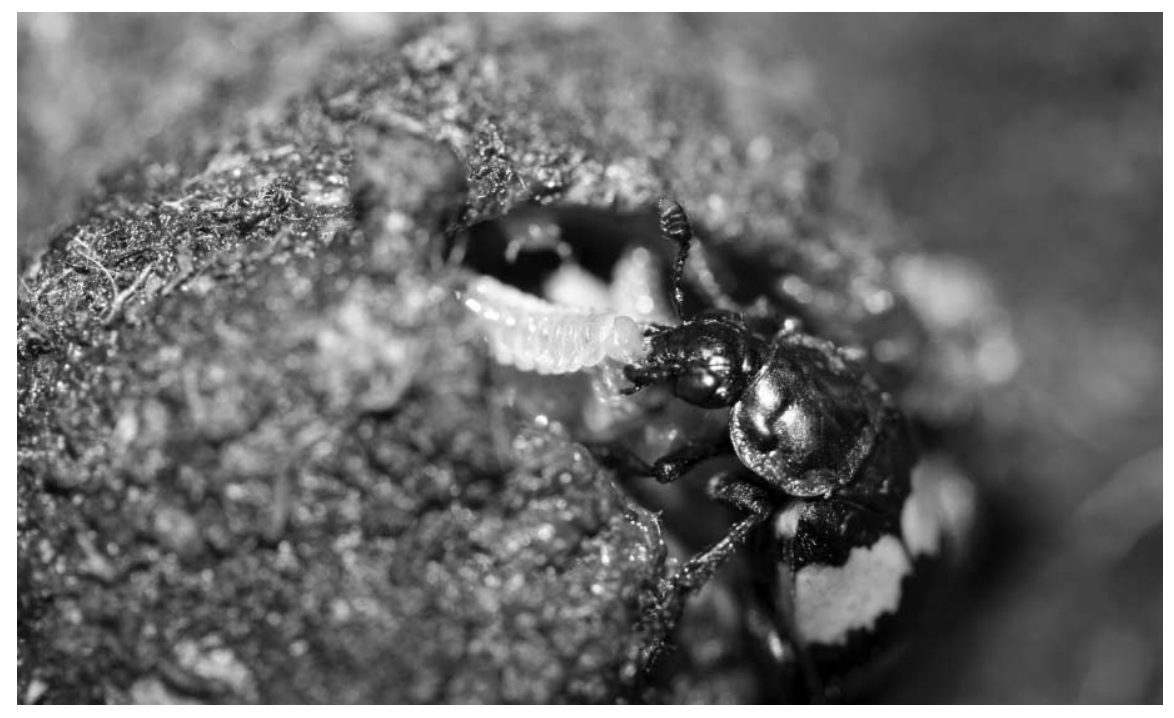

Burying beetle mother feeding a larva (photograph by Allen J. Moore).

Associate Editor: Locke Rowe Editor: Michael C. Whitlock 